\title{
La Organización Mundial del Comercio (OMC) y la reducción del espacio para la política: El caso argentino ante el resurgi- miento de la restricción externa durante la posconvertibilidad.
}

\author{
The World Trade Organization (WTO) and the re- \\ duction in policy space: The resurgence of the exter- \\ nal constraint in Argentina during the post-convert- \\ ibility regime.
}

Juan Manuel Padin²

\begin{abstract}
Resumen: La creación de la OMC a mediados de los años noventa limitó el espacio para la política comercial. Un ejemplo concreto de esta situación lo ofrecen los reducidos márgenes de acción con los que contó la Argentina para administrar el flujo de divisas por la vía comercial ante la reaparición de la restricción externa a partir del año 2011. En tal sentido, el presente trabajo se propone problematizar los alcances de las reglas de la OMC y evaluar las alternativas que ofrece esta organización para enfrentar una situación crítica en la balanza de pagos a partir del análisis del caso argentino.
\end{abstract}

Palabras clave: OMC; Balance de Pagos; Restricción externa; Posconvertibilidad

Abstract: The birth of the WTO in the mid1990s shrank the policy space. A clear example of this was the narrowed range of policies still available to manage foreign currency flows through trade channels before the reappearance of the external constraint in 2011 in Argentina. This paper, then, aims to question the scope of the WTO rules and to evaluate the options offered by such organization to face a critical balance of payments deficit, basing the analysis on the argentine case.

Keywords: WTO; Balance of Payments; External Constraint; Post Convertibility

\footnotetext{
DOI: https://doi.org/10.24215/23142766e056

${ }^{1}$ Recibido: 26/10/2018. Aceptado: 21/05/2019

${ }^{2}$ Licenciado en Ciencia Política (Universidad de Buenos Aires), Magíster en Economía (Facultad Latinoamericana de Ciencias Sociales), Diplomado en Desarrollo Local y Economía Social (Facultad Latinoamericana de Ciencias Sociales), Doctorando en Desarrollo Económico (Universidad Nacional de Quilmes). Email: jmanuelpadin@gmail.com
} 


\section{Introducción}

Las transformaciones que se produjeron en la configuración de la economía mundial desde los años setenta bajo la óptica de un capitalismo de libre mercado originaron presiones a efectos de impulsar un proceso de apertura comercial a nivel global. Este resultado alcanzó su cenit en los años noventa y se cristalizó en múltiples iniciativas (unilaterales, bilaterales, regionales y multilaterales) a través de las cuales se consolidó un entramado normativo más permeable al libre flujo de mercancías y servicios en la vasta mayoría de las naciones del mundo.

En el caso de los países latinoamericanos, la liberalización comercial fue solo una faceta de un proceso de apertura económica más amplio que se concretó decididamente en la década del noventa con el auspicio del Consenso de Washington y con el aliento tanto del Banco Mundial (BM) como del Fondo Monetario Internacional (FMI), los que facilitaban el acceso a créditos en tanto se adoptaran determinadas políticas económicas (privatizaciones, ajuste fiscal, apertura financiera y reformas laborales, entre otras).

El papel de estos organismos internacionales fue muy relevante a la hora de impulsar la aplicación de políticas de corte neoliberal. No obstante, en lo que respecta a la apertura comercial, fue otra institución la que operó como soporte multilateral de esta agenda, impulsando un agudo rediseño de las reglas por las que se rige el comercio internacional. Nos referimos a la Organización Mundial del Comercio (OMC), creada el 1 de enero de 1995 como producto de la Ronda de Negociaciones de Uruguay, la cual logró ampliar el alcance original del Acuerdo General sobre Aranceles Aduaneros y Comercio de 1947 (GATT de 1947) más allá del comercio de mercancías, lo que incluía, entre otras cuestiones, el comercio de servicios, los derechos de propiedad intelectual y las medidas en materia de inversiones relacionadas con el comercio.

Los compromisos asumidos por los miembros de esta organización limitaron el espacio para la política comercial -entendido aquí como la habilidad de actuar o el margen de acción disponible con que cuenta un gobierno para aplicar políticas-, a cambio de un (supuesto) mejor acceso a los mercados y un positivo desempeño comercial. Estos límites, sin embargo, tuvieron un claro impacto diferencial. La firma del Acuerdo sobre la OMC prohibió o limitó el uso de ciertos instrumentos utilizados históricamente por los países desarrollados (en adelante, PD) y los países de industrialización reciente ${ }^{3}$. Estas opciones, en consecuencia, dejaron de estar al alcance de los países en desarrollo (en adelante, PED) ${ }^{4}$, independientemente de la situación social que éstos enfrentasen y de su apremio por transformar sus estructuras económicas, condición indispensable para ofrecer una mejor calidad de vida a sus ciudadanos.

Un ejemplo concreto de lo antedicho lo ofrece la Argentina en la posconvertibilidad.

\footnotetext{
${ }^{3}$ En muchos casos, las prohibiciones o restricciones no estaban ligadas a cambios normativos sino que ya eran parte del legado del GATT de 1947. Pero el refuerzo de los mecanismos de cumplimiento, junto a aclaraciones o modificaciones en determinadas disposiciones, robustecía su operatividad.

${ }^{4}$ Más precisamente, el fortalecimiento de un sistema basado en reglas aumentó el riesgo de sufrir represalias ante la implementación de medidas incompatibles con los compromisos asumidos. Por supuesto, ser parte del sistema también otorga beneficios que explican, en gran medida, las razones por las cuales la mayoría de los países pertenece a una organización como la OMC.
} 
La administración kirchnerista (2003-2015), a partir de una revigorizada política de administración del comercio exterior ante la reaparición de la restricción externa ${ }^{5}$ en 2011 , implementó una serie de iniciativas con vistas a evitar el deterioro de la balanza de pagos. Esta modalidad de intervención generó fuertes reacciones en gran parte del núcleo empresarial y alertó a varios gobiernos extranjeros, los cuales decidieron solicitar el establecimiento de un grupo especial al Órgano de Solución de Diferencias (OSD) de la OMC, alegando que las medidas aplicadas eran incompatibles con las reglas de la OMC.

Desde la óptica del derecho de la OMC, no obstante, resultaba jurídicamente posible para la Argentina invocar las disposiciones vigentes a efectos de "salvaguardar la situación financiera exterior y obtener un nivel de reservas suficiente para la ejecución de un programa de desarrollo económico". Pero, por diversas cuestiones jurídicas y extra-jurídicas que presentaremos a continuación, el camino elegido fue otro.

En razón de lo expuesto, el artículo se propone problematizar los alcances de las reglas de la OMC y hacer una evaluación crítica de las alternativas existentes en el Organismo para enfrentar una situación apremiante en la balanza de pagos a partir del análisis del caso argentino, a la luz de dos hipótesis: i) que estas reglas, si bien prevén mecanismos para lidiar con este tipo de escenarios, incluyen un conjunto de condicionantes que reducen su relevancia en términos prácticos; y ii) que la persistencia de inconsistencias macroeconómicas en el país generó presiones sobre la política comercial con vistas a intentar apaciguar por esa vía los desbalances generados, lo cual dio paso a un doble efecto: al mismo tiempo que se constriñó el espacio para la política disponible, se tornó aún más complejo invocar los mecanismos de la OMC teniendo en cuenta la amplitud que tuvo el control estatal sobre las importaciones.

Con estos objetivos, el trabajo se estructura del siguiente modo luego de esta breve introducción. En primer lugar, se destaca el rol e incidencia de la OMC respecto al espacio para las políticas (policy space). Luego, en la tercera sección, se busca analizar el margen que ofrecen las disposiciones de la OMC para intervenir ante una situación crítica en la balanza de pagos, para lo cual se desarrolla el concepto de "restricción externa", subrayando su relevancia histórica en la Argentina. En la cuarta sección, se aborda el resurgimiento de la restricción externa en el país durante la posconvertibilidad y se detallan las medidas adoptadas por el gobierno kirchnerista, así como las derivaciones del caso que enfrentó la Argentina en la OMC a solicitud de Estados Unidos, la Unión Europea y Japón. Por último, se presentan las reflexiones finales.

\section{La OMC y el espacio para la política}

Cuando un gobierno firma un acuerdo comercial, es esperable que obtenga una serie de beneficios a cambio de comprometerse a tomar ciertas medidas, como la reducción de las barreras arancelarias y no arancelarias al comercio. Es en este marco que los países se comprometen a limitar su espacio para la política, entendido aquí como la "habilidad de

\footnotetext{
${ }^{5} \mathrm{La}$ "restricción externa" hace referencia a la recurrente falta de disponibilidad de divisas que demanda la economía en su fase ascendente. Este concepto, que da cuenta de una cuestión de índole estructural en la Argentina, se desarrolla con mayor detalle en el apartado 4.1.
} 
actuar" o como "el universo de opciones de acción gubernamental para lograr un determinado objetivo de política pública" (Bosch, 2009:120).

Es dable advertir que la limitación del margen de acción no es un tema novedoso; en efecto, siempre es un punto presente (y en tensión) en cualquier negociación. La relación de los imperios con sus colonias o semicolonias, y/o entre países soberanos pero con relaciones marcadamente asimétricas, ofrece múltiples ejemplos históricos. También existen casos de ampliación del espacio para la política, como lo evidenciaron muchos PED entre finales de la segunda guerra y la crisis del petróleo de los años setenta, momento a partir del cual se redujo -en términos generales- dicha tendencia, especialmente al finalizar la guerra fría (Chang, 2005a). Lo relevante, en todo caso, es evaluar la naturaleza y alcance de la reducción (o ampliación) del espacio, y cómo esta puede afectar a un país en desarrollo como la Argentina- en cierta coyuntura histórica. Para esto, resulta indispensable justipreciar la dimensión normativa (incluida la arquitectura, el alcance y el contenido específico de las disposiciones de los acuerdos) a la luz de los beneficios esperados e indagar si lo acordado permite o no, y de qué modo, alcanzar los objetivos que determina cada sociedad a través de los mecanismos institucionales establecidos para tal fin.

Históricamente, los PD y los países de industrialización reciente han hecho uso de un conjunto de herramientas para impulsar el desarrollo que desde mediados de los años 1990, y como consecuencia de la firma del Acuerdo sobre la OMC, se encuentran vedados o seriamente limitados. Esto marca un contrapunto con lo acontecido durante la vigencia del GATT de 1947, cuando el incumplimiento de la normativa multilateral era comúnmente pasado por alto. De acuerdo a Di Caprio y Gallagher (2006), el GATT funcionaba básicamente como un foro para que los países industriales negociaran concesiones arancelarias. En el caso de los PED, la predominancia de esta lógica generaba un doble efecto: por un lado, no era usual que se incluyeran sus preocupaciones en este ámbito; pero, por el otro, tampoco se aplicaba una vigilancia activa sobre sus políticas nacionales (ni acerca de su coherencia con los compromisos multilaterales). No obstante, la situación comenzó a modificarse en los años ochenta, cuando los programas de ajuste estructural del Banco Mundial y el Fondo Monetario Internacional luego de la crisis de la deuda latinoamericana de 1982-comenzaron a reducir el espacio para la política hasta entonces existente (Chang, 2005a).

El punto de quiebre en la esfera comercial multilateral, de todos modos, comenzó a forjarse en 1986 con el inicio de la mentada Ronda Uruguay (1986-1994). Este avance regulatorio multilateral dio lugar a un nuevo régimen de política que expandió su alcance ${ }^{6}$ y fortaleció los mecanismos de cumplimiento de las regulaciones. Lo antedicho resultó prontamente evidenciado por aquellos miembros que continuaron controlando celosamente ciertas importaciones, exigiendo requerimientos de contenido local, creando (o manteniendo) esquemas impositivos cuya finalidad subyacente era proteger la industria nacional, o ejerciendo una débil vigilancia sobre los derechos de propiedad intelectual.

La preocupación de los PED respecto a las consecuencias de los acuerdos abarcados sobre su margen de acción salió a la luz a pocos años del inicio de las actividades de la OMC, en consonancia con el estallido de sucesivas crisis financieras desde mediados de los años 1990. En este contexto, durante el proceso preparatorio de la Conferencia Ministerial de Seattle (Estados Unidos, 1999), la delegación de Venezuela realizó un planteó que consti-

\footnotetext{
${ }^{6}$ Los temas comercial que abarca la OMC se detallan en https://www.wto.org/spanish/tratop_s/tratop_s.htm
} 
tuyó un hito en el debate sobre el "espacio para las políticas" (policy space). El país sudamericano subrayaba la necesidad de darle un contenido sustantivo a la "dimensión del desarrollo" que fuese más allá de la concesión de períodos de transición y la provisión de asistencia técnica a los PED, con la finalidad de que éstos pudieran ajustarse a lo dispuesto en los acuerdos abarcados. La propuesta de Venezuela fue el punto de partida para que un conjunto de países e intelectuales le dieran vida a una valiosa discusión acerca de las consecuencias que podía acarrear la normativa de la OMC en el caso de los PED.

Una segunda fase de este debate, que podríamos denominar de "diseminación", se produjo entre la Conferencia Ministerial ${ }^{7}$ de Seattle (1999) y la Conferencia Ministerial de Cancún (2003) ante la pretensión -fallida- de Estados Unidos, la UE y Japón de ampliar la agenda de compromisos hacia nuevas esferas (como las compras públicas, inversiones, competencia y facilitación del comercio). Esto hubiese implicado una reducción adicional del espacio para políticas, razón por la cual varios PED, intelectuales y organizaciones (como la UNCTAD y la CEPAL) aunaron fuerzas y lograron incluir estos temas en la agenda internacional. Desde esos años a la actualidad, el debate perdió la intensidad inicial que marcó sus comienzos, pero fue ampliándose hacia nuevos campos, incluidas las investigaciones sobre el espacio para la política en disciplinas específicas (subsidios, agricultura, propiedad intelectual, etc.), la utilización histórica de estos por parte de los PD y PED, su impacto en ciertos sectores económicos, o su relevancia en las negociaciones comerciales bilaterales o regionales.

De acuerdo a Corrales (2007), en esta discusión se pueden distinguir dos grandes posicionamientos. Por un lado, aquellos que hacen hincapié en la necesidad de flexibilidades para las políticas de desarrollo por el lado de la oferta (creación de capacidades y diversificación productiva, entre otras) sin renunciar a los compromisos de liberalización comercial y buscando combinar ambos planos para una inserción positiva de los PED en la economía internacional. Y por otro lado, se destacan los académicos que debaten respecto a la liberalización comercial y sus consecuencias. Aquí encontramos dos subgrupos: el primero propone limitar la liberalización y expandir la flexibilidad para erigir barreras comerciales mientras que el segundo desacredita esta idea, señalándola como un intento de volver al proteccionismo a fin de restaurar políticas de sustitución de importaciones.

La tarea de interpretar la reducción del espacio para la política ha contado con el aporte de numerosos autores de distintas vertientes teóricas. Es posible distinguir, en términos generales, tres posiciones diferenciadas. En primer lugar, aquellos que consideran que el impacto no es tan gravoso en el contexto del desarrollo y que los acuerdos abarcados permiten la utilización de una amplia gama de instrumentos de política (Amsden, 2001; Amsden e Hikino, 2000; Rodrik, 2004; Bora et al. 2000; Pangestu, 2002; Akyüz, 2008; Weiss, 2006). En segundo lugar, hay quienes entienden que la reducción del espacio sí es sustancial y limita seriamente el universo de posibilidades disponibles para los PED (Abugattas y Paus, 2006; Chang, 2005a, 2005b; Cimoli et al., 2008; Cuello, 2004; Kumar et al., 2007; Lall, 2004; Wade, 2003). En tercer y último lugar, los autores que enfatizan la necesidad de ajustarse a las disposiciones de la OMC dado que alejarse de las reglas del sistema multilateral solo puede producir un daño (autoinfligido) para el miembro que tome ese camino (Kleen y Page, 2005; Messerlin, 2007).

\footnotetext{
${ }^{7}$ Sobre las características y estructura de la Organización, ver https://www.wto.org/spanish/thewto s/thewto s.htm
} 
Vale destacar en este punto que, como parte del desarrollo conceptual sobre el "espacio para las políticas", varios intelectuales se enfocaron simultáneamente en examinar cuáles son los elementos o dimensiones que restringen o amplían el "espacio realmente existente", más allá de la dimensión jurídica. Aquí se destacan, en primer término, las "restricciones endógenas", las que incluyen el tamaño del país y su disponibilidad de recursos económicos, financieros y humanos; las capacidades institucionales; y la aceptabilidad de ciertas políticas por parte de actores clave. En segundo término, cobran relevancia las "restricciones exógenas", las que comprenden, entre otros elementos, el entramado de compromisos internacionales, los efectos de la globalización -el mercado capitalista global y el comportamiento de sus principales actores- sobre la soberanía nacional y las opciones de política, y el impacto de medidas aplicadas por terceros en el país analizado 8 .

De igual forma, otros autores enfatizan el rol que pueden tener ciertos factores en la reducción del "espacio efectivo". Chang (2005a) menciona que, además del papel de los acuerdos internacionales, se deben incluir las amenazas de salidas de capital, el rol de las agencias calificadoras, las organizaciones internacionales y los medios de comunicación. Akyuz (2008b) se refiere al impacto de las políticas de apertura, liberalización y desregulación, y al papel de las instituciones de Bretton Woods; y Lall (2004) resalta que las políticas de ajuste estructural y las presiones de los países ricos, junto a los acuerdos bilaterales de inversión y comercio y la normativa de la OMC, restringen la habilidad de los gobiernos para ejecutar políticas industriales.

En conclusión, más allá de las posiciones respecto del impacto que tuvo el entramado normativo de la OMC en las posibilidades de aplicar ciertas medidas de política, es evidente que ser miembro de la OMC tuvo (y tiene) consecuencias diferenciales de acuerdo a los efectos derivados del tipo de restricciones que afronta cada Miembro. La Argentina, por supuesto, no constituye una excepción a esta regla.

\section{La restricción externa y las disposiciones OMC en materia de Ba- lance de Pagos}

El concepto de "restricción externa" se relaciona con la falta de disponibilidad de divisas para acceder a los insumos y bienes de capital que demanda la economía en su fase ascendente. Este asunto reviste gran actualidad a pesar de que, en la Argentina, históricamente estuvo asociado al proceso de industrialización sustitutiva de importaciones (19301975) y actuó como limición al crecimiento sostenido de la actividad económica durante períodos prolongados (Prebisch, 1986; Braun, 1970; Diamand, 1972).

Las causas subyacentes de este fenómeno durante la industrialización sustitutiva de importaciones (ISI) estaban directamente vinculadas a una estructura productiva desequilibrada compuesta por dos sectores (el sector primario y el sector industrial), donde el primero se destacaba por su mayor productividad respecto al segundo. En este marco, el crecimiento económico - motorizado por la industria-requería cantidades crecientes de divisas que no podían ser obtenidas por este sector, en tanto su (alto) nivel de precios le impedía

\footnotetext{
${ }^{8}$ Esta cuestión es tratada particularmente en Hamwey, 2005; Abugattas y Paus, 2006; Mayer, 2009; y Shadlen, 2005a y $2005 b$, entre otros.
} 
exportar. El sector agropecuario era entonces el encargado de proveer los recursos necesarios para garantizar un crecimiento sostenible de la tasa de actividad, pero su pobre desempeño sectorial no le permitía cumplir este rol. Así, la divergencia entre el crecimiento industrial (demandante de divisas) y el sector agropecuario (proveedor) se convertía en responsable de la crisis de balanza de pagos en la Argentina.

Esta situación, que se manifestaba críticamente a través del canal comercial (déficit comercial), se modificó a finales de los años 1970, cuando los flujos internacionales de capital permitieron diferir sus consecuencias pero no sortearlas. En efecto, durante la ISI el comportamiento de la balanza de pagos era determinado, esencialmente, por el desempeño de la cuenta corriente, ya que la cuenta capital tenía un papel secundario (Schteingart, 2016). Pero esto empezó a modificarse como corolario del endeudamiento externo que se produjo durante la Dictadura Militar (1976-1983), en un contexto internacional de liquidez en el cual los flujos financieros se redirigieron hacia América Latina.

En las décadas siguientes, el fenómeno de la restricción fue examinado desde diferentes perspectivas que dan cuenta tanto de su evolución como del peso que adquirieron otros factores como fuentes de egreso de divisas. Entre ellos, sobresalen además de la singular trayectoria de la balanza comercial dadas las características de la estructura productiva, el creciente giro de utilidades y dividendos ante el peso determinante del capital extranjero y la concentración económica (Schorr y Wainer, 2014), la fuga de capitales (Basualdo, 2006; Gaggero et al., 2013), el impacto generado por el flujo volátil de capitales que se produce en la periferia (Barbosa Filho, 2002), y otros elementos que merecen ser destacados dada su relevancia en ciertas coyunturas, como el saldo negativo de la balanza energética o la salida de divisas vinculada al turismo.

El fenómeno descripto, por supuesto, excede largamente a la Argentina. Como ejemplo, cabe recordar que el propio GATT de 1947 receptaba esta preocupación en su entramado normativo brindando alternativas, desde la política comercial, para aquellos países que enfrentasen situaciones críticas en la balanza de pagos. Estas alternativas jurídicas, que consistían en la aplicación de restricciones a las importaciones de mercaderías, sufrieron algunas modificaciones a lo largo de los años, pero siguen formando parte del entramado normativo vigente en la actualidad ${ }^{9} \mathrm{y}$ definen con cierto detalle en qué circunstancias es posible invocar estas disposiciones y qué conjunto de compromisos se deben asumir ante tal eventualidad.

En este sentido, cuando un miembro aplica restricciones (o aumenta las existentes), a efectos de salvaguardar su balanza de pagos, debe notificar al Consejo General de la OMC y también está en la obligación de entablar Consultas (plenas o simplificadas) con el Comité de Restricciones por Balanza de Pagos ${ }^{10}$. En los procedimientos de Consulta plena, que se realizan anualmente y que aplican los países desarrollados y los países en desarrollo -con

\footnotetext{
${ }^{9}$ Nos referimos, específicamente, a los artículos XII (invocado por los PD) y XVIII: B (invocado por los PED) del GATT de 1994, la "Declaración sobre las Medidas Comerciales adoptadas por motivos de balanza de pagos de 1979", y el "Entendimiento relativo a las disposiciones del GATT de 1994 en materia de Balanza de Pagos".

${ }^{10}$ Este Comité es el encargado de informar al Consejo General lo analizado en el marco de las Consultas, incluidas las propuestas de recomendaciones tendientes a promover la aplicación del entramado normativo vigente. El Consejo también puede formular recomendaciones al miembro que aplica las restricciones.
} 
excepciones-, también participa el Fondo Monetario Internacional, el cual aporta documentación y realiza una declaración formal. En el caso de los procedimientos de Consulta simple, que cuentan con una periodicidad bianual y utilizan los países menos adelantados y determinados países en desarrollo (siempre que estén realizando esfuerzos de liberalización, de conformidad con un calendario convenido con el Comité en una consulta anterior, o que hayan sido objeto de un examen de sus políticas comerciales en el mismo año civil), el FMI no efectúa una declaración formal.

El papel del Fondo Monetario y el rol que debe tener tanto el Comité de Restricciones por Balanza de Pagos como el Consejo General, cabe alertar, ha despertado fuertes polémicas. Un claro ejemplo de lo antedicho se presentó en la diferencia denominada "DS90: India - Restricciones cuantitativas a las importaciones de productos agrícolas, textiles e industriales". En 1997 la India conservaba restricciones cuantitativas sobre 2.714 líneas arancelarias. El país había notificado las medidas al Comité de Restricciones de Balanza de Pagos y efectuado las consultas de rigor, ocasión en la cual propuso eliminar las restricciones en un plazo de siete años. En dicha oportunidad varios países, entre los cuales se encontraba Estados Unidos, consideraron abusiva la propuesta, al tiempo que solicitaron iniciar consultas con el país asiático.

La India llegó a un acuerdo con la mayoría de los Miembros que participaron inicialmente de este mecanismo (Australia, Canadá, las Comunidades Europeas, Nueva Zelandia y Suiza) con la excepción de Estados Unidos, que solicitó la conformación de un Grupo Especial al Órgano de Solución de Diferencias de la OMC. En este marco, la ex colonia británica argumentó que el Grupo que intervenía en la disputa no tenía autoridad para determinar si el calendario presentado para el retiro de las restricciones a las importaciones estaba en consonancia con el artículo XVIII: $\mathrm{B}^{11}$ y que la autoridad competente en este asunto era el Comité de Restricciones por Balanza de Pagos y el Consejo General. También planteó que las conclusiones a las que llegó el FMI sobre la balanza de pagos de la India no podían ser aceptadas como dispositivas, puesto que tal escenario supondría otorgarle a este organismo internacional la prerrogativa de determinar el estatus jurídico de una restricción bajo el Artículo XVIII.

El Grupo Especial que examinó el caso, sin embargo, consideró que las reservas indias no eran insuficientes, basándose en los informes del Banco de la Reserva de la India y del FMI, y determinó que no había justificación alguna para las restricciones cuantitativas aplicadas por la India. Por otra parte, acompañó el argumento institucional de Estados Unidos con respecto a que el Órgano de Solución de Diferencias sí tenía autoridad para intervenir en el caso.

El resultado de esta disputa marcó un hito en relación con la utilización de las restricciones a las importaciones bajo el Artículo XVIII: B. De acuerdo a McKuscker (2000), el caso sepultó una era en la cual los PED podían utilizar la ambigüedad del lenguaje del Acuerdo para proteger sus industrias nacionales. Pero también, como secuela adicional, alertó a otros Miembros respecto del impacto que podía tener la participación del FMI. En consecuencia, algunos países alzaron la voz manifestando su "preocupación acerca de varios aspectos de las consultas recientes celebradas en el Comité de Restricciones por Balanza de

\footnotetext{
${ }^{11}$ El texto del artículo puede ser consultado en el sitio oficial de la OMC: https://www.wto.org/spanish/docs_s/legal_s/gatt47_02_s.htm. Para mayores referencias sobre la temática, ver:

https://www.wto.org/spanish/tratop_s/bop_s/bop_s.htm
} 
Pagos y de la solución de diferencias en esta esfera, en particular: la función desempeñada por el FMI en el proceso de consultas y el carácter de su aportación, a la luz del mandato enunciado en el artículo XV del GATT. EI FMI no debía hacer suya la función del Comité de Restricciones por Balanza de Pagos. La aportación del FMI debía ser analítica y no descriptiva, en particular cuando formula observaciones sobre políticas nacionales que sólo habían tenido un efecto indirecto en la balanza de pagos o cuando fija calendarios para la eliminación de las restricciones comerciales" (OMC, 2002: 3). La India, por su parte, también manifestó su disconformidad en diversos foros, señalando que una dimensión crítica, como lo es la dimensión del desarrollo, había quedado relegada a partir de la creación de la OMC.

A pesar de las intensas críticas que suscitó el caso, muchos de los temas planteados quedaron sin resolución por falta de consenso entre los miembros, entre ellos, cuestiones de relevancia institucional, como la definición de la competencia exclusiva del Comité de Restricciones por Balanza de Pagos para examinar la justificación de las medidas; o la realización de un examen integral del artículo a efectos de "facilitar el desarrollo progresivo de las economías de los países en desarrollo y permitirles ejecutar sus programas y aplicar sus políticas de desarrollo económico tendientes al aumento del nivel de vida general de su población" 12 .

De todas maneras, debemos subrayar que los cambios acaecidos desde los años setenta, a partir del colapso de los acuerdos de Bretton Woods, se reflejaron en una declinación en el uso de la política comercial para influenciar variables macroeconómicas. Estos cambios consistieron en el desmantelamiento de las regulaciones sobre los movimientos de capitales; una fuerte internacionalización de los grandes bancos multinacionales en un marco donde se experimentó un proceso de fragmentación e internacionalización de los procesos productivos guiado por menores costos de producción en las diferentes regiones del mundo y por la articulación de una red de contratistas y subcontratistas a nivel regional o mundial; y el impulso de la demanda de recursos por parte de los Estados Nacionales (vía endeudamiento externo), que también fue acompañada por grandes firmas mediante la emisión de títulos y obligaciones (Santarcángelo, 2017).

Ante el nuevo escenario económico global, Hoekman y Kosteki (2013) afirman que las disposiciones en trato tenían mayor sentido durante la vigencia del sistema de tipos de cambio fijos de Bretton Woods, pero desde los años 1970 resultaba más efectivo recurrir a un ajuste en el tipo de cambio y a la disciplina fiscal y monetaria para enfrentar desbalances en la cuenta corriente. En contraposición, varios intelectuales apuntaron a la OMC y al FMI, y señalaron la importancia de repensar las medidas disponible relativas a la balanza de pagos tomando en cuenta los desafíos que enfrentan los PED al llevar a cabo su política económica (Siegel, 2002).

En resumidas cuentas, podemos concluir que el recurso de las restricciones a las importaciones de mercancías por motivos de balanza de pagos dista de ser un camino simple. La invocación de los artículos mencionados supone lidiar con el FMI en un marco donde, entre otras cuestiones, no están claramente delineadas las competencias del Consejo Gene-

\footnotetext{
12 La cita pertenece al documento JOB(01)/152/Rev.1 [Recapitulación de cuestiones pendientes relativas ala aplicación planteadas por los Miembros]. En el documento JOB (05)/60, por otra parte, se describe el estado de situación de las consultas sobre cuestiones pendientes relativas a la aplicación respecto a los temas vinculados a las disposiciones en materia de balanza de pagos.
} 
ral y del Comité de Restricciones por Balanza de Pagos, el método de estimación de suficiencia de reservas no toma en cuenta la dimensión del desarrollo, los plazos para la eliminación de medidas suelen ser puestos en cuestión, el alcance del articulado de las disposiciones no es claro, y subsisten ciertas zonas grises en los procedimientos (CEI, 2015). A su vez, el invocar este mecanismo no exime a un miembro del riesgo de terminar dirimiendo estas cuestiones en el Órgano de Solución de Diferencias.

\section{La modalidad de intervención del Kirchnerismo ante el resurgi- miento de la restricción externa}

\subsection{La reaparición de la restricción externa en la posconvertibilidad y la modali- dad de intervención del Kirchnerismo}

Por varios años, la restricción externa parecía haber sido condenada al ostracismo. Durante la Convertibilidad, por ejemplo, y mientras se lograron garantizar flujos financieros que compensen el déficit comercial y la pérdida de divisas por pago de utilidades y dividendos, la restricción permaneció latente ${ }^{13}$. En el decenio comprendido entre el año 2000 y 2009, su amenaza parecía diluirse en tanto el superávit comercial fungía como sostén del saldo positivo de la cuenta corriente, pero las cosas empezarían a modificarse a partir del año 2010: luego de ocho años consecutivos de superávit, la cuenta corriente cambiaba de signo. Aun manteniéndose el superávit comercial, éste ya no lograba compensar el incremento del déficit en servicios y, particularmente, en rentas, donde el giro de utilidades y dividendos había adquirido mayor relevancia (superando los 10.000 millones de dólares desde 2010). La restricción externa, indudablemente, había vuelto a escena y ocupaba el primer plano.

El proceso de pérdida de reservas internacionales que se desató a partir de 2011 nos brinda una idea aproximada de la magnitud del problema: mientras que en 2010 el país contaba con 52 mil millones de dólares de reservas, éstas alcanzaban los 27 mil millones de dólares en marzo de 2014. Sobre este punto, Schteingart (2016:52) explica que "a partir de 2010-2011, el proceso de acumulación de reservas absolutas llegó a un máximo, y el del stock relativo entró en una etapa de pronunciada contracción: de un monto equivalente al $102 \%$ de los pagos de importaciones, intereses y utilidades en 2009 se pasó a apenas el 30\% en 2013."

Al respecto, debemos destacar que la sinuosa trayectoria que marcó a la economía argentina en tal periodo se produjo en un escenario de apreciación del tipo de cambio real ${ }^{14}$ que sumó presión al sector externo ${ }^{15}$. Esto resulta evidente al examinar el índice del tipo de

\footnotetext{
${ }^{13}$ Sin embargo, a partir de 1998 estuvo cada vez más presente hasta el estallido mismo del plan de Convertibilidad en 2001.

${ }^{14}$ Canitrot (1983) problematiza, en particular, las tensiones que produce la apreciación del tipo de cambio real en las cuentas externas.

${ }^{15}$ Es preciso señalar que en enero de 2014 tal situación, sumada a las presiones del mercado, condujo a una devaluación relevante.
} 
cambio real multilateral elaborado por el Banco Central de la República Argentina ${ }^{16}$ : mientras que en octubre de 2009 su valor era de 151,6 para el mes de noviembre de 2013 se encontraba en 98,6 . Luego, se produjo un incremento, con un nuevo pico $(114,8)$ como producto de la devaluación de enero de 2014. No obstante, desde allí y hasta prácticamente el final del mandato de Cristina Fernández, se evidenció un fuerte descenso, hasta alcanzar un piso de 75,4 en noviembre de 2015.

En este marco, otros factores redoblaron las tensiones. Entre éstos, sobresalen la caída de los términos de intercambio ${ }^{17}$, la crisis energética (que se tradujo en un déficit sectorial superior a los 2 mil millones de dólares en 2012 y a los 6 mil en 2013) y el fuerte déficit de otros sectores como el automotriz y bienes de capital. Así, como corolario, mientras que el saldo comercial entre los años 2003 y 2012 fue de 14 mil millones de dólares -promedio anual-, éste cayó considerablemente entre 2013 y 2014 (4.600 y 6.000 millones). Por otra parte, la cuenta corriente también registraba salidas de divisas provenientes de la cuenta turismo y el incremento del giro de utilidades, que superó los 9,8 mil millones de dólares en promedio- entre 2010 y 2013, a lo que se agregaba la fuga de capitales ${ }^{18}$.

La salida de divisas de la economía nacional encendió las alarmas del gobierno kirchnerista, que recurrió a múltiples medidas con la finalidad de evitar la caída sistemática de reservas y sortear una crisis del sector externo que afectase negativamente el nivel de actividad y empleo. Las medidas implementadas desde el inicio de la restricción externa incluyeron una intervención en planos múltiples donde además de la devaluación gradual desde los últimos meses de 2011, se buscó tanto incrementar la afluencia de divisas como restringir su salida, potenciando los controles sobre las operaciones que impacten sobre ellas ${ }^{19}$.

En este punto, nos interesa detenernos en particular en las políticas de índole comercial vinculadas a las importaciones de bienes. Se destaca aquí, como medida paradigmática, la obligación de presentar una Declaración Jurada Anticipada de Importaciones (DJAI) antes de realizar una compra. Este requisito estaba asociado a otros requerimientos que debían ser contemplados, en la gran mayoría de los casos, por las firmas de mayor envergadura. Entre estos, se incluía: i) el equilibrio de la balanza comercial por firma, ii) la limitación del volumen de importaciones, iii) el compromiso de congelar o reducir precios en el mercado

\footnotetext{
${ }^{16}$ Base 17-12-15=100, disponible en https://www.bcra.gob.ar/Pdfs/PublicacionesEstadisticas/ITCRMSerie.xls

${ }^{17}$ El índice de términos de intercambio (base 2004=100, INDEC) pasó de un pico de 151 el 4 to trimestre de 2012 a un piso de 121 el 2do trimestre de 2015.

${ }^{18}$ Gaggero et al. (2013) señalan la formación de activos en el exterior como otro elemento clave en la reaparición de la restricción externa: en 2011, superó los 25 mil millones de dólares.

${ }^{19}$ Entre las iniciativas que llevó a cabo la administración kirchnerista y que marcaron el pulso de esta etapa de la política económica, sobresalen el "blanqueo" de capitales, el cambio en la integración mínima diaria en moneda extranjera de los bancos, los swaps de monedas con otros países, el acuerdo con el Club de París y el pago a firmas multinacionales con laudos favorables en el CIADI a fines de salir al mercado de capitales, la regulación del mercado de cambios, las limitaciones al giro de utilidades, los recargos a las operaciones que involucren pagos en moneda extranjera, la pesificación de pensiones extranjeras, mayores controles sobre las casas de cambio, los límites a la compra de bienes vía correo postal, la presión a los grandes exportadores para que liquidasen sus stocks -especialmente del complejo sojero- y la creación de un seguro de cambio virtual que los indujera a igual fin, y la reducción de los tiempos para el ingreso de divisas por exportación al sistema financiero local.
} 
argentino como condición para importar; ; iv) la incorporación de contenido local, v) la realización de inversiones en el país, y/o no girar utilidades.

Las DJAI, cabe recordar, habían sido establecidas en el mes de febrero de 2012 mediante las Resoluciones Generales N 3252 y N 3255 de la AFIP. A través de este instrumento se fijaba, como requisito previo a la importación, el envío de información anticipada (datos del importador, posición arancelaria, unidades a importar, montos comprometidos, origen y procedencia de la mercadería, entre otros). Esa información era evaluada por diferentes organismos, entre los que se destacaba la Secretaría de Comercio. En función de tal análisis, se autorizaba (o no) la correspondiente operación. Los requerimientos complementarios aludidos previamente, por otra parte, eran fijados a través de acuerdos, generalmente informales, que alcanzaba el Ministerio de Industria y/o la Secretaría de Comercio con las principales empresas que operaban en el país.

Es preciso advertir, finalmente, que el fuerte control sobre las importaciones de bienes que conllevó esta medida se llevó a cabo no sólo en un escenario interno signado por fuertes desequilibrios, sino también en un contexto internacional precedido por la crisis de $2008^{20}$, que había llevado a los miembros del G20, por ejemplo, a alcanzar el compromiso de no implementar medidas restrictivas del comercio ${ }^{21}$. La reacción de Cristina Fernández de Kirchner frente a las dificultades, no obstante, fue un giro hacia un desarrollismo más nacionalista, que a su vez daba cuenta de que, si a principios de los años 90 había una sintonía entre el modelo de desarrollo de la Argentina y la ideología de la OMC, luego de la crisis internacional se produjo un rotundo cambio de escenario (Zelicovich, 2016: 57).

\subsection{El caso "Argentina-Medidas que afectan a la importación de mercancías"}

La nueva administración del comercio ensayada por la Argentina dio paso a una solicitud de consultas en la OMC y el posterior establecimiento de un Grupo Especial ante el OSD a pedido de la Unión Europea, Estados Unidos y Japón, durante el transcurso de 2012. En particular, el caso denominado "Argentina-Medidas que afectan a la importación de mercancías" reconoce como primer antecedente formal la solicitud de consultas realizada por la Unión Europea el 25 de mayo de 2012, que impugnaba las DJAI, la utilización de las licencias de importación y ciertos requisitos comerciales adicionales aplicados por el país, denominados prescripciones relativas al comercio (PRC). Luego, se sumaron reclamos similares en las solicitudes de consultas cursadas por Estados Unidos y Japón (fechadas ambas el 21 de agosto de 2012) y México (presentada el 24 de agosto de 2012). Este último país, de todos modos, decidió no avanzar con la disputa luego de que prosperaran negociaciones bilaterales ${ }^{22}$.

Al no resolverse las diferencias en la etapa de consultas, los reclamantes solicitaron el

\footnotetext{
${ }^{20}$ Es preciso remarcar, siguiendo a Stancanelli (2009), la magnitud de la crisis, en tanto se trataba de la crisis de mayor importancia después de la registrada en los años treinta del siglo pasado, potenciada por los diagnósticos divergentes entre los principales países respecto a su naturaleza (y los pasos a seguir para solucionarla).

${ }^{21} \mathrm{Al}$ respecto, ver G20 (2014).

${ }^{22}$ El 21 de noviembre de 2012 retiró su solicitud de establecimiento de un grupo especial del orden del día de la reunión del OSD prevista para el 17 de diciembre de 2012. En este resultado fueron decisivas las negociaciones vinculadas al intercambio automotriz entre ambos países, regulado por el Acuerdo de Complementación Económica $\mathrm{N}^{\circ} 55$.
} 
establecimiento de sendos Grupos Especiales, ante lo cual el OSD decidió en su reunión del 28 de enero de 2013 establecer un único Grupo para examinar los asuntos planteados ${ }^{23}$, que se conformó el 27 de mayo de 2013.

En las presentaciones de los demandantes se cuestionaban aspectos intrínsecos del régimen de DJAI como, por ejemplo, la falta de información del alcance de las observaciones que podían formularse, el procedimiento a seguir para conocer cuáles eran las observaciones, la forma de cumplimentar esas observaciones, o los motivos de intervención de las distintas agencias gubernamentales. También se indicaba que las DJAI reemplazaban, en esencia, a las licencias no automáticas (LNA) pero con un alcance mucho mayor ${ }^{24}$. Una vez cumplidas todas las instancias procesales, el Grupo Especial encargado de examinar el caso ${ }^{25}$ presentó su informe el 22 de agosto de 2014, en donde concluyó, finalmente, que la Argentina había actuado de manera incompatible con los artículos XI:1 y III:4 del GATT de $1994^{26}$. El Grupo adicionalmente consideró que la medida PRC tenía efectos limitativos en la importación de mercancías en la Argentina, además de resaltar su falta de transparencia y previsibilidad, lo cual desalentaba aún más las importaciones. En el caso de las DJAI, se determinó que el procedimiento DJAI tenía un efecto limitativo en las importaciones y, por lo tanto, también constituía una restricción. En línea con estas apreciaciones, el Grupo recomendó que el Órgano de Solución de Diferencias solicitase a la Argentina poner "las medidas incompatibles en conformidad con las obligaciones que le corresponden en virtud del GATT de 1994". Luego, como el informe fue apelado, se dio lugar a la intervención del OA, que finalmente emitió sus informes el 15 de enero de 2015, los que fueron adoptados por el OSD en su reunión de 26 de enero de 2015.

Ante este resultado ${ }^{27}$, la Argentina expresó su voluntad de aplicar las recomendaciones y resoluciones del OSD y solicitó un plazo prudencial de aplicación, que fue acordado de

\footnotetext{
${ }^{23}$ Arabia Saudita, Australia, Canadá, China, Corea, Ecuador, Estados Unidos, Guatemala, India, Israel, Japón, Noruega, Suiza, Tailandia, el Taipei Chino, y Turquía, reservaron sus derechos en calidad de terceros.

${ }^{24}$ Las DJAls no estaban limitadas a las posiciones arancelarias cubiertas por las LNA, sino a todas las posiciones de una parte sustancial de los subregímenes aduaneros de importación con ciertas excepciones, entre ellas, los regímenes de donaciones, muestras, franquicias diplomáticas, courier y envíos postales.

${ }^{25}$ Para realizar este examen se contempla, lógicamente, el análisis técnico de las medidas en cuestión (el entendimiento sobre las medidas en sí, sus características y funcionamiento) y la evaluación jurídica respecto a su encuadramiento, a efectos de juzgar su eventual incompatibilidad con las disposiciones aludidas en la disputa.
}

${ }^{26}$ El Artículo XI, relativo a la eliminación general de las restricciones cuantitativas, reza en su primer inciso que: "Ninguna parte contratante impondrá ni mantendrá -aparte de los derechos de aduana, impuestos u otras cargas- prohibiciones ni restricciones a la importación de un producto del territorio de otra parte contratante o a la exportación o a la venta para la exportación de un producto destinado al territorio de otra parte contratante, ya sean aplicadas mediante contingentes, licencias de importación o de exportación, o por medio de otras medidas." El Artículo III.4, por su parte, afirma que: "Los productos del territorio de toda parte contratante importados en el territorio de cualquier otra parte contratante no deberán recibir un trato menos favorable que el concedido a los productos similares de origen nacional, en lo concerniente a cualquier ley, reglamento o prescripción que afecte a la venta, la oferta para la venta, la compra, el transporte, la distribución y el uso de estos productos en el mercado interior. Las disposiciones de este párrafo no impedirán la aplicación de tarifas diferentes en los transportes interiores, basadas exclusivamente en la utilización económica de los medios de transporte y no en el origen del producto."

${ }^{27}$ Zelicovich (2016:60) manifiesta que “...en el plano doméstico la presentación de esta diferencia generó rechazo en el gobierno de Fernández de Kirchner, fortaleciéndose aquellas lecturas del orden internacional, 
común acuerdo con los reclamantes, con fecha de expiración el 31 de diciembre de $2015^{28}$. La última instancia del caso concluyó el 14 de enero de 2016, cuando el país notificó que había cumplido plenamente las recomendaciones y resoluciones del OSD al dejar de aplicar las prescripciones relacionadas con el comercio y derogar las DJAI.

De este modo, se dio por finalizado el conflicto que desató, originariamente, el intento de enfrentar la restricción externa recurriendo, entre otras cuestiones, a la aplicación de ciertas medidas comerciales. Es importante subrayar, de todos modos, que entre la solicitud de consultas y la derogación de las medidas pasaron casi tres años y medio. El plazo transcurrido, en este caso, jugó a favor de un miembro PED.

La derogación de las DJAI se produjo, de todos modos, en un marco muy diferente signado por el cambio de orientación del estado nacional a partir del triunfo en las elecciones de Mauricio Macri en 2015, que abrió una nueva etapa de relacionamiento con las potencias demandantes ${ }^{29}$.

Resulta evidente en este punto que el resurgimiento de la restricción externa enfrentó a la administración kirchnerista a un duro desafío, ante el cual tomó un camino no exento de riesgos. El caso descripto nos muestra que si se recurre a la política comercial limitando las importaciones sin invocar el Artículo XVIII, los senderos son estrechos: ya no resulta posible desplegar ciertas medidas con el doble propósito de administrar el flujo de divisas y proteger la producción nacional. Al menos, no sin arriesgarse a sufrir un duro revés en la $\mathrm{OMC}^{30}$.

Esta lectura, de todos modos, es eminentemente parcial si no recordamos además del antecedente indio y las cuestiones problemáticas no resueltas respecto al Artículo XVIII, la tensa relación existente entre el Kirchnerismo y el FMI, que convertía a la invocación de este artículo en una opción prácticamente inviable en términos políticos. La cancelación de la deuda con el FMI, la negativa a someterse a la evaluación prevista en el Artículo IV del Convenio Constitutivo de este organismo y la polémica en torno a la confiabilidad de las estadísticas públicas conformaban antecedentes de peso para rechazar esta alternativa.

Invocar el Artículo XVIII:B, por otra parte, hubiese instituido una nota disonante en el frente interno: después de negar tensiones respecto a la disponibilidad de divisas y acusar al FMI en diversos foros recordando el resultado de sus recetas, ¿el Gobierno argentino sostendría en la OMC que precisaba salvaguardar su situación financiera exterior y se sometería a la evaluación de un representante del FMI en el Comité de Restricciones por Balanza de Pagos?

según las cuales la globalización es asumida como una amenaza y donde las instituciones existentes reproducen un orden injusto y restringen las posibilidades de desarrollo de los países menos poderosos."

${ }^{28} \mathrm{Al}$ respecto, ver WT/DS438/23. https://docs.wto.org/dol2fe/Pages/FE_Search/FE_S_S006.aspx?Query=(@Symbol=\%20wt/ds438/*)\&Language=SPANISH\&Context=FomerScriptedSearch\&languageUIChanged=true

${ }^{29}$ Zelicovich (2018: 56) sostiene que "Macri implementó una reestructuración del sistema de Declaraciones Juradas Anticipadas de Importación (DJAI), adoptando en su lugar un "Sistema integral de Monitoreo de las Importaciones" (SIMI), el cual se erigió en una combinación de licencias automáticas y no automáticas de importación con muchas menos posición es arancelarias afectadas."

30 Por otra parte, como sostiene Félix Peña (2015: 22), para restringir las importaciones "también se requiere contar con un grado de organización eficiente en las instituciones gubernamentales del comercio exterior y negociaciones comerciales internacionales y, en especial, se necesita contar con funcionarios incluyendo los del mayor nivel jerárquico- con un buen entendimiento de las normas de la OMC y con mucha experiencia práctica." 


\section{Conclusiones}

Luego de más de dos décadas transcurridas desde la creación de la OMC, es claro que las negociaciones de la Ronda Uruguay tuvieron un impacto considerable en los espacios para la política de los PED. La reducción del margen de acción disponible de estos países, a su vez, suele verse potenciada mediante la acción de otros factores adicionales, definidos en la literatura especializada como restricciones "endógenas" y "exógenas", las cuales afectan de modo diferencial a cada uno de los miembros de la organización.

Siguiendo esta perspectiva, en el presente artículo detallamos -a la luz de las disposiciones OMC- las acciones desplegadas en el ámbito comercial por la administración kirchnerista ante la reaparición de la restricción externa y afirmamos que el reducido espacio para la política descripto en el trabajo reconoce como una de sus causas a los conflictos (y antecedentes) derivados de las regulaciones del comercio internacional (Artículo XVIII:B) vinculadas a la intervención de los países miembro ante una delicada situación en la balanza de pagos. Aquí se destacan tanto elementos intrínsecos a su articulado y procedimientos asociados, como lo evidencia la experiencia de la India, como la relación que tenía en su momento la Argentina con el FMI y el papel establecido para este organismo en dicha disposición. Lo antedicho avala nuestra primera hipótesis de trabajo, en el sentido de que las reglas que regulan el comercio internacional, si bien prevén mecanismos particulares para lidiar con este tipo de escenarios, incluyen un conjunto de condicionantes que reducen su relevancia en términos prácticos.

En igual sentido, consideramos que la experiencia de la Argentina también expresa los estrechos límites con los que cuentan los PED si se corren de las opciones previstas en la normativa multilateral y los riesgos que esto conlleva si otros miembros deciden recurrir al Órgano de Solución de Diferencias. En este punto también es importante remarcar, de modo complementario, el rol que tuvo la crisis internacional como elemento subyacente en el caso "Argentina - Medidas que afectan a la importación de mercancías", ya que condujo a un incremento de las presiones de los países centrales contra los países de la periferia que oponían resistencia a la libre circulación de mercancías, donde la disputa con la Argentina también fungía como advertencia velada para el resto de los países miembro que intentasen replicar (o ya estuvieran llevando a cabo) este tipo de prácticas.

Entre las "restricciones endógenas" que constriñeron todavía más el espacio disponible para la política en el caso argentino, encontramos varios factores que coadyuvaron a la conformación de un cuadro de situación sumamente complejo. En particular, nos referimos a la apreciación del tipo de cambio, la caída de los términos de intercambio y varias dificultades adicionales reseñadas que signaron los últimos años de la administración kirchnerista. En este escenario, la restricción externa terminó desatando renovadas tensiones hacia todos los vértices de política vinculados con operatorias que incidiesen en el saldo de divisas. En tal orden, resulta evidente que tratar de apaciguar por la vía política comercial los efectos de las inconsistencias macroeconómicas distorsionó dicha vía ${ }^{31}$, alejando (todavía más) cualquier intento de invocar el entramado normativo multilateral, tal como sostuvimos en la segunda hipótesis de trabajo.

\footnotetext{
${ }^{31}$ Delich (2014), por su parte, señala que la incompatibilidad de las medidas con las reglas de la OMC es una dimensión menor del problema, y lo central (para el bienestar de los argentinos) es que sean o no efectivas para el problema que intenta solucionar un gobierno recurriendo a éstas.
} 
Por otra parte, la fuerte administración del comercio exterior deterioró la (ya) conflictiva relación con ciertos actores económicos, como el capital extranjero y los grupos económicos locales, que no compartían la orientación de políticas del gobierno, en particular en los últimos años de la administración kirchnerista. A esta situación, se sumaba el fuerte enfrentamiento con los medios de comunicación, lo cual hacía más compleja la defensa pública de estas iniciativas y le brindaba elementos adicionales a los reclamantes para la descripción del marco bajo el cual se llevaban a cabo las medidas impugnadas luego ante el Organismo de Solución de Diferencias.

Otros factores de naturaleza endógena también resultaban notorios, aunque se destaquen principalmente por su ausencia, entre ellos, la falta de un adecuado entramado institucional que permita llevar adelante una política comercial más precisa y evite (o suavice) eventuales embates a nivel nacional e internacional, que pongan en riesgo legítimos objetivos de política. Al respecto, la modalidad de intervención del Gobierno ante esta coyuntura no se destacó, ciertamente, por su "pericia quirúrgica", lo cual elevaba consecuentemente el riesgo de sufrir un fallo adverso a nivel multilateral. No obstante, de todos modos, debemos señalar que dado el margen existente en el Acuerdo de la OMC sin invocar el Artículo XVIII, difícilmente una administración comercial intensa cuyo objetivo primordial sea el de contribuir a evitar una situación externa crítica pueda evitar (o sortear exitosamente) una instancia como la del Órgano de Solución de Diferencias de la OMC. No obstante, y a pesar del resultado del caso, por el cual la Argentina debió derogar las medidas impugnadas, el Gobierno sí consiguió un tiempo prudencial (más de 3 años), al haber aprovechando al máximo las diversas etapas procedimentales que conllevan los litigios en la OMC.

El resultado finalmente alcanzado, de todos modos, donde sobresalen más allá del fallo de la OMC, la pérdida de reservas en una economía signada por una sinuosa trayectoria y la derrota electoral en $\mathbf{2 0 1 5}$ que dio paso a un radical cambio de orientación política y económica, convoca necesariamente a repensar alternativas. Dentro de estas podría alegarse con toda razón que, dada la naturaleza estructural de la restricción externa en la Argentina, reformar el Artículo XVIII:B dotándolo de mayor flexibilidad, debería ser un mandato permanente de los funcionarios argentinos en la agenda multilateral. Sin embargo, aquí es preciso advertir que el hecho de que esto no haya sucedido no refleja necesariamente desazón institucional o falta de voluntad: los mecanismos político-institucionales de la OMC, las relaciones políticas (donde tallan fuertemente las potencias), y el poder de lobby que ejercen los principales grupos económicos a nivel global, expectantes a efectos de moldear la normativa multilateral, actúan como obstáculos quijotescos a la hora de repensar alternativas favorables a los PED respecto a la regulación del comercio internacional.

Explorar otros caminos haciendo uso de diversas medidas de política comercial externa reconoce reducidos contornos. En efecto, no existe demasiado margen, una vez que la restricción externa se encuentra en vigencia, para implementar iniciativas compatibles con los compromisos multilaterales que permitan detener (o suavizar eficazmente) una crisis del sector externo. Empero, a pesar de que muchas veces estos fenómenos globales aparecen como una suerte de fuerzas irrefrenables de mercado contra las que no se puede hacer nada, existe un pequeño margen de maniobra dentro de las regulaciones multilaterales que puede ser fructífero si se toman -a tiempo- las decisiones de economía política adecuadas.

De todos modos, tampoco se debe sobredimensionar el rol de la balanza comercial sobre la restricción externa ni el papel de la política comercial externa a efectos de lidiar con sus 
consecuencias. Como vimos, con diversos los factores que operan sobre ella (la propia configuración de la estructura productiva, la fuga de capitales, el giro de utilidades, el endeudamiento externo, etc.), con intensidad variable de acuerdo a la coyuntura histórica. Operar sobre cada uno de ellos conduce a la utilización de diversos instrumentos que exceden, lógicamente, el campo de acción de la política comercial externa. En tal sentido, tratar de modificar aquellos aspectos de los acuerdos que hayan sido identificados como limitantes estratégicos utilizando, a su vez, el espacio disponible parecería ser la opción más sensata.

La concreción de esta política, sin embargo, requiere de un enorme esfuerzo enmarcado en una estrategia político-económica donde se defina, entre otros ejes, el tipo de inserción comercial deseada. Esta decisión no puede estar divorciada de la evolución del resto de los factores constitutivos de una política de desarrollo como lo son, a modo de ejemplo, la política macroeconómica, industrial, tecnológica, energética, o fiscal. De lo antedicho deriva necesariamente la importancia de contar con mayores capacidades estatales que permitan encarar un desafío de esta magnitud.

En conclusión, contar con mejores herramientas es imperativo a efectos de enfrentar la restricción externa. La política comercial es una de las dimensiones que puede contribuir en ese orden ${ }^{32}$ y esta contribución puede ser mayor si se logra, a nivel multilateral, impulsar una agenda destinada a ampliar el espacio para políticas de los miembros PED que contemple instrumentos más acordes a sus necesidades. Esta es sino solo una parte de la solución, la cual debe ser acompañada, entre otras cuestiones, por una política comercial que se encuentre comprendida dentro de un proyecto político-económico de desarrollo que logre articular sus múltiples ejes constitutivos y que contemple, asimismo, los recursos necesarios para la construcción de capacidades acordes a este desafío.

\section{Bibliografía}

Abugattas y Paus (2006). "Policy Space for a Capability-Centered Development Strategy for Latin America”. En: Diego Sanchez Ancochea and Kenneth Shadlen (ed.) The Political Economy of the Hemispheric Integration. Responding to Globalization in the Americas. England: Palgrave Macmillan.

Akyüz (2008) Global Rules and Markets: Constraints over Policy Autonomy in Developing Countries. Geneva: International Labour Office.

Amsden, A. (2001). The rise of "the rest". Challenges to the West from Late-Industrializing Economies. England: Oxford University Press.

Amsden, A. H., \& Hikino, T. (2000). "The Bark Is Worse Than the Bite: New WTO Law and Late Industrialization." The ANNALS of the American Academy of Political and Social Science, 570(1), 104-114. https://doi.org/10.1177/000271620057000108

\footnotetext{
${ }^{32}$ Sobre este asunto, Bouzas y Cabello (2017: 204) entienden que es necesario introducir cambios operativos y organizacionales en la forma en que se implementa y diseña a política comercial, así como aumentar la transparencia del proceso de formulación de política, eliminar la duplicación de esfuerzos y recursos existentes en este campo, y mejorar la calidad del apoyo técnico de que goza el sector público y el que puede brindar el sector privado.
} 
Barbosa Filho, N. (2002). "The Balance-of-payments Constraint: From Balanced Trade to Sustainable Debt", Working Paper, núm.2001.06, Nueva York, Schwartz Center for Economic Policy Analysis (SCEPA), New School University.

Basualdo, E. (2006). Estudios de historia económica. Desde mediados del siglo XX a la actualidad. Buenos Aires: Siglo XXI Editores.

Bora, B., Lloyd, P. \& Pangestu, M. (2000). Industrial Policy and the WTO. UNCTAD, Policy Issues in International Trade and Commodities, Study Series No. 6.

Bosch, Roberto (2009). "Las negociaciones comerciales y la reducción del espacio para políticas para el desarrollo industrial." Revista del CEI, número 14, p. 117 a 131.

Bouzas, R. y Cabello, S. (2007). "La Formulación de la Política Comercial en la Argentina: Fundamentos Estructurales e Institucionales de la Volatilidad". En: Marcos Sawaya Jank / Simão Davi Silber. Políticas Comerciais Comparadas: Desempenho e Modelos Organizacionais. San Pablo: Editorial Singular.

Braun, O. (1970). "Desarrollo del capital monopolista en la Argentina." En: O. Braun (compilador). El capitalismo argentino en crisis. Buenos Aires: Siglo XXI.

Canitrot, A. (1983). "El salario real y la restricción externa de la economía." Revista Desarrollo Económico. Volumen $\mathrm{N}^{\circ} 23, \mathrm{~N}^{\circ} 91$.

CEI (2015). Momento de redefiniciones en el Sistema Multilateral de Comercio: ¿oportunidad para recuperar las herramientas de política industrial perdidas? Iniciativa "Friends of Industrialization".

Chang, H. (2005a). "Policy Space in Historical Perspective - with special reference to Trade and Industrial Policies", conferencia dictada en Tufts University.

Chang, H. (2005b) Why Developing Countries need tariffs? How WTO NAMA negotiations could deny developing countries' right to a future? South Centre, Geneva, and Oxfam International, Oxford.

Mario Cimoli \& Giovanni Dosi \& Joseph E. Stiglitz. (2008). "The Future of Industrial Policies in the New Millennium: Toward a Knowledge-Centered Development Agenda," LEM Papers Series 2008/19, Laboratory of Economics and Management (LEM), Sant'Anna School of Advanced Studies, Pisa, Italy.

Corrales, W. (2007). "Una perspectiva de América Latina y el Caribe sobre los espacio para políticas en las estrategias de desarrollo sostenible apoyadas en el comercio." Documento de Proyecto. Comisión Económica para América Latina y el Caribe, Naciones Unidas.

Cuello, F. (2004). “¿Es Posible Preservar los Espacio para políticas de Desarrollo en la Organización Mundial del Comercio?" FES/CIECA, (Ed.) Desarrollo y Políticas Comerciales. Buenos Aires: Fundación Friedrich Ebert Stiftung.

Delich, V. (2014). “Multilateralismo, incertidumbre y reputación. Las disputas argentinas en la Organización Mundial del Comercio". En: Donadio Linares (coord.). Controversias en la Organización Mundial del Comercio. Protagonismo y estrategias de los países en desarrollo. Buenos Aires: Editorial Teseo.

Gallagher, Kevin \& Dicaprio, Alisa. (2006). “The WTO and the Shrinking of Development 
La Organización Mundial del Comercio (OMC) y la reducción del espacio para la política (...) (77- 96)

Space." The Journal of World Investment \& Trade. 7. 781-803.

Diamand, M. (1972). "La estructura productiva desequilibrada argentina y el tipo de cambio." Desarrollo Económico. Disponible en: http://www.jstor.org/stable/3465991

G20 (2014): "Boosting trade for growth and jobs", G20-Australia Policy Note. Disponible en: http://www.g20australia.org/sites/default/files/g20 resources/library/Trade\%20policy\%20note.pdf

Gaggero, J. Rúa, M. y Gaggero, A. (2013). “Argentina. Fuga de Capitales (2002-2012)”. IADE.

Hamwey, R. (2005) Expanding national policy space for development: Why the multilateral trading system must change. Working Paper 25. South Centre.

Hoekman, B. \& Kostecki, M. (2013). The Political Economy of the World Trading System: WTO and Beyond. 3rd Edition. Oxford: OUP.

Kleen, P. \& Page, S. (2005). "Special and Differential Treatment of Developing Countries in the World Trade Organization." Global Development Studies No. 2, Ministry for Foreign Affairs Sweden.

Kumar, N. \& Gallagher, R. (2007). "Relevance of 'Policy Space' for Development: Implications for Multilateral Trade Negotiations." RIS-DP \#120. Research and Information System.

Lall, A. (2013). "Reinventing Industrial Strategy: The Role Of Government Policy In Building Industrial Competitiveness." Annals of Economics and Finance, Society for AEF, vol. 14(2), pages 785-829, November.

Mayer, J. (2009). "Policy Space: What, for What, and Where?" Development Policy Review, 2009, 27 (4): 373-395.

McKuscker, K. (2000). "Are Trade Restrictions to Protect the Balance of Payments Becoming Obsolete?” INTERECONOMICS, March/April 2000.

Messerlin, P. (2007). "Enlarging the Vision for Trade Policy Space: Special and Differentiated Treatment and Infant Industry Issues". En: Greenaway, D (ed.) The World Economy, Global Trade Policy, 1395-1407. Malden, MA: Blackwell Publishing.

OMC (2002). Documento WT/BOP/W/24. Comité de Restricciones por Balanza de Pagos. Labor realizada sobre las cuestiones relativas a la aplicación de conformidad con el párrafo 12 b) de la Declaración de Doha. Informe al Comité de Negociaciones Comerciales.

Pangestu, M. (2002). "Industrial Policy and Developing Countries." En: Bernard Hoekman, Aaditya Mattoo, and Philip English (ed.). Development, Trade and WTO. A Handbook. The International Bank for Reconstruction and Development / The World Bank.

Peña, F. (2015). "El fallo de la OMC sobre medidas comerciales de Argentina: consideraciones y perspectivas." Revista Puentes, Volumen 16, Número 12, bbril de 2015.

Prebisch, R. (1986) "El desarrollo económico de la América Latina y algunos de sus principales Problemas". Desarrollo Económico, Vol. 26, No. 103.

Rodrik, A. (2004). "Industrial policy for the twenty-first century". KSG Working Paper No. 
RWP04-047.

Santarcángelo, J. (2017). “Las transformaciones de la economía mundial y el endeudamiento de América latina." Revista Voces del Fénix, № 64 (Deuda Externa).

Schorr, M. y Wainer, A. (2014). "Trabas a la acumulación capitalista en un país dependiente: La restricción externa al crecimiento en la Argentina de posconvertibilidad." VIII Jornadas de Sociología de la UNLP, 3 al 5 de diciembre de 2014, Ensenada, Argentina. En Memoria Académica.

Schteingart, D. (2016) “La restricción externa en el largo plazo: Argentina, 1960-2013.” Revista Argentina de Economía Internacional. Número 5. Febrero de 2016.

Shadlen, K. (2005a). "Exchanging Development for Market Access? Deep Integration and Industrial Policy under Multilateral and Regional-Bilateral Trade Agreements." Review of International Political Economy, Vol. 12, No. 5 (Dec., 2005), pp. 750775.

Shadlen, K. (2005b). "Policy Space for Development in the WTO and Beyond: The Case of Intellectual Property Rights." Global Development and Environment Institute. Working Paper N. 05-06.

Siegel, D. (2002). "Legal aspects of the IMF/WTO relationship: the fund's articles of agreement and the WTO agreements." The American Journal of International Law. Vol. 96:561-621.

Stancanelli, N. (2009). “La crisis económica internacional y el papel del G20." Revista del CEI N 14.

Wade, R. (2003). "What strategies are viable for developing countries today? The World Trade Organization and the shrinking of 'development space'." Review of International Political Economy, 10(4), 621-644.

Weiss, L. (2006). “Gobernanza global, estrategias nacionales: cómo los estados hacen espacio para desenvolverse bajo la OMC." Versión revisada de la ponencia presentada en la Conferencia Internacional sobre la Política y las Políticas La Política y las Políticas en un mundo que se globaliza, 24-26 de julio, 2003, Bristol University.

Zelicovich, J. (2016). “Políticas comerciales, participación y caprendizajes? de Argentina en el vigésimo aniversario de la OMC". En: Delich, V., Lopez, D. y Muñoz, F. (2016). 20 años de la OMC: una perspectiva desde Latinoamérica. Buenos Aires: FLACSO. Sede Académica Argentina, Programa de Cátedras OMC, Universidad de Chile.

Zelicovich, J. (2018). “Claves y tensiones de la estrategia argentina de política comercial externa en la búsqueda de una 'inserción inteligente al mundo' (2015-2018)". Latin American Journal of Trade Policy 2 (2018) - ISSN 079-9668 - Universidad de Chile. 